\title{
Correlation between Analgesic Effect of Electroacupuncture "ST36" - "GB34" and DNA Methyltransferase on Sciatica Rats Dnmt3a
}

\author{
Qiu-ling XU ${ }^{1,2, a,{ }^{*}}$ and Tao LIU ${ }^{1,3, b}$ \\ ${ }^{1}$ Hainan Medical University, Haikou 571101, China \\ ${ }^{2}$ Doctor, professor. Research direction: Neurobiological mechanism of \\ acupuncture. \\ ${ }^{3}$ Professor, Research direction: Neurobiological mechanism of \\ acupuncture. \\ axq17809@163.com \\ b13647549720@163.com \\ ${ }^{*}$ Corresponding author
}

Keywords: Acupuncture analgesia, DNA methyltransferase, Dnmt3a.

\begin{abstract}
To observation the relationship between spinal inhibition of DNA methylation in electroacupuncture (EA) analgesia, and spinal cord Dnmt3amRNA expression. EA and inhibitors of 5-AZA Dnmt can improve CCI animals hyperalgesia state and decreased Dnmt3amRNA expression. DNA methylation is closely related to chronic pain, acupuncture ST36, GB34 can relieve pain, and can regulate Dnmt3a.
\end{abstract}

\section{Introduction}

In recent years, studies have shown that [1], closely related to chronic pain and epigenetics. Epigenetics refers to the DNA sequence does not change but the gene expression is heritable changes have taken place, mainly reflected in DNA methylation, histone modification, non-coding RNA regulation, gene imprinting, $\mathrm{X}$ chromosome inactivation.DNA methylation is the most thorough research in vertebrate epigenetic mechanisms. Acupuncture analgesia has a long history, zusanli (ST36), yanglingquan (GB34) is commonly used in the clinical treatment of sciatica acupoints. In this experiment to sciatic nerve Chronic strain injuries (Chronic Constrictive Injury, CCI) model as the carrier, to explore the relationship of the electroacupuncture "ST36" - "GB34" and DNA methyltransferase Dnmt3a on sciatica rats. 


\section{Materials}

A total of 40 clean-class, male Wistar rats, weighing $180-200 \mathrm{~g}$, were purchased from Experimental Animal Center of Guangzhou.

\section{Methods}

\section{Establishment of Chronic Neuropathic Pain (CCI) Model}

Under anesthesia, the rat's left sciatic nerve was exposed at the mid-thigh level by blunt dissection through the biceps femoris muscle. Four constrictive ligatures (4-0 surgical suture) were tied around the nerve at the distal end close to the nerve bifurcation at spaces about 1.0 $\mathrm{mm}$ apart.

\section{Electroacupuncture}

Bilateral Zusanli (ST 36) and Yanglingquan (GB 34) were punctured with filiform needles (Gauge 28), respectively, and electrically stimulated using a HANS EA Apparatus (LH202, Beijing Huawei Industrial Developing Company, Beijing, China). EA (2/15 Hz, $1 \mathrm{~mA})$ intervention was administered for 30 minutes, once daily beginning from the $4^{\text {th }}$ day on after CCI surgery for 10 days for rats of the CCI+EA group.

\section{Thermal Pain Threshold Detection}

With RB - 200 intelligent hot plate apparatus (chengdu Tai union technology co., LTD.)Paw withdrawal latency (i.e., pain threshold) of the bilateral footplates was measured three times with an interval of 3-5 minutes between detections. To avoid potential tissue damage, the cutoff time of the radiant heat radiation was set to 20 seconds. Thermal pain threshold detection was conducted prior to, as well as 3,7,10 and 14 days after CCI.

\section{Real Time Quantitative PCR Method to Detect the Expression of Dnmt3a:}

After the CCI surgery14 days, taking out the lumbar intumescence spinal cord segment the specific primers DNMT3a as follows: the upstream Primer (5'- 3') GGCCCATTCGATCTGGTGA, downstream primers (5'-3') CTTGGCTATTCTGCCGTGTTC,the result is expressed and standardization of Dnmt3a gene.

\section{Statistical Analysis}

Data were expressed as mean \pm SD. Differences in paw withdrawal latency were assessed using one-way analysis of variance with 
repeated measures when appropriate. Least significant difference was used to compare data between every two groups. A value of $P<0.05$ was considered statistically significant.

\section{Results}

\section{EA decreased Pain Reaction after CCI}

In the CCI (by ligature of the left sciatic nerve) rat groups, pain scores [paw withdrawal latency (PWL) of the healthy limb - PWL of the affected limb] were significantly bigger than in the normal control group $(\mathrm{P}<0.05)$, suggesting a decreased pain threshold. The pain scores were significantly less in the CCI + EA group and CCI + 5AZA group than in the CCI group, respectively $(\mathrm{P}<0.05)$. In comparison with the CCI + EA group, the pain score of the CCI + 5AZA group was significantly higher $(P<0.05)$, (Table 1$)$.

Table 1. Comparison of the pain scores among rats of the control, chronic constrictive injury (CCI), CCI+5AZA ,CCI + electroacupuncture (EA) groups ( $\bar{x} \pm \mathrm{s}, \mathrm{sec}, 10 \mathrm{rats} /$ group).

\begin{tabular}{|c|c|c|c|c|c|}
\hline \multirow{2}{*}{$\begin{array}{l}\text { GRO } \\
\text { UPS }\end{array}$} & \multirow[t]{2}{*}{ Base HAS } & \multicolumn{4}{|c|}{ Days after surgey } \\
\hline & & 3 & 7 & 10 & 14 \\
\hline Control & $0.25 \pm 0.02$ & $0.24 \pm 0.04$ & $0.24 \pm 0.05$ & $0.23 \pm 0.04$ & $0.25 \pm 0.06$ \\
\hline CCI & $0.23 \pm 0.03$ & $2.45 \pm 0.21 \mathrm{a}$ & $3.02 \pm 0.23 \mathrm{a}$ & $3.31 \pm 0.13 a$ & $\begin{array}{l}2.45 \pm 0.12 \\
\mathrm{a}\end{array}$ \\
\hline $\begin{array}{c}\mathrm{CCI}+5 \\
\mathrm{AZA}\end{array}$ & $0.24 \pm 0.02$ & $2.33 \pm 0.07 \mathrm{a}$ & $2.54 \pm 0.15 \mathrm{a}$ & $2.5 \pm 0.14 \mathrm{a}$ & $\begin{array}{l}2.04 \pm 0.28 \\
\mathrm{ab}\end{array}$ \\
\hline $\begin{array}{c}\mathrm{CCI}+\mathrm{E} \\
\mathrm{A}\end{array}$ & $0.25 \pm 0.03$ & $2.4 \pm 0.34 \mathrm{a}$ & $2.67 \pm 0.18 \mathrm{a}$ & $2.73 \pm 0.11 \mathrm{a}$ & $\begin{array}{l}1.7 \pm 0.29 \\
\mathrm{ab} \mathrm{c}\end{array}$ \\
\hline
\end{tabular}

\section{EA decreased Dnmt3a mRNA Expression}

Compared with the control group, the Dnmt3a mRNA express of CCI group was significantly higher $(\mathrm{P}<0.05)$, suggesting increased Dnmt3a, Dnmt3b mRNA expression after CCI. In comparison with the CCI group, the Dnmt3a expression was considerably decreased in the CCI+EA group $(\mathrm{P}<0.05)$ rather than in the CCI+5AZA group, suggesting the EA and 5AZA can decrease the Dnmt3a expression increased after CCI. Compared to the CCI+5AZA group, the Dnmt3a mRNA expression was reduced also, but $(\mathrm{P}>0.05$, Table 2$)$. 
Table 2. Comparison of Dnmt3a mRNA expression among rats of the control, CCI, $\mathrm{CCI}+5 \mathrm{AZA}, \mathrm{CCI}+\mathrm{EA}$ groups.

\begin{tabular}{ccc}
\hline GROUPS & CASE & DNMT3A \\
\hline Control & 10 & $1.47 \pm 0.05$ \\
CCI & 10 & $1.59 \pm 0.04 \mathrm{a}$ \\
CCI+5AZA & 10 & $1.53 \pm 0.07 \mathrm{ab}$ \\
CCI+EA & 10 & $1.52 \pm 0.04 \mathrm{ab}$ \\
\hline
\end{tabular}

$\mathrm{aP}<0.05$, vs. the normal control group; $\mathrm{b} \mathrm{P}<0.05$, vs. the $\mathrm{CCI}$ group; $\mathrm{c} \mathrm{P}<0.05$, vs. the CCI+5AZA group.

\section{Discussion}

Studies have shown that [2], DNA methylation transferase (DNMT) inhibitors 5 - azacytidine can inhibit DNA methylation level and the expression of DNMT, achieve the result that relieve pain. DNA methyltranferase (DNMT) inhibitor 5-Aza-2'-deoxycytidine significantly reduced incision-induced mechanical allodynia and thermal sensitivity [3]. Abzianidze E [4]found that during acute inflammatory pain induced by the application of $10 \%$ mustard oil on the tongues of rats, levels of DNMT3a and $3 \mathrm{~b}$ were elevated markedly (36 and $42 \%$ respectively), whereas the level of DNMT1 was not changed significantly. Their data suggest that NSAIDs (alone or in combination with DNMT inhibitors) may be proposed as possible epigenetic regulatory agents, which may play a role in epigenetic mechanisms indirectly through altering the activity of inflammatory mediators involved in pain development.This experiment rats intrathecal injection of Dnmt inhibitors 5 - AZA, can significantly reduce the CCI rats pain, in accordance with the above research resultsWang Y[5] found that Up-regulation of DNMTs in the lumbar spinal may play an important role in the pathogenesis ofpathogenesis of neuropathic pain in CCI rats. Pollema-Mays SL [6] suggest that DNMT regulation in adult DRGs may be a contributor to the pain phenotype and merits further study.

GB34 and ST36 is the most commonly used two points in the treatment of sciatica.GB34 is foot shaoyang bile of the acupuncture point, and also the "jin" of eight will point. Acupuncture GB34 have the effect that relaxes the muscles and strong muscle.GB34 acupoints local anatomy as phil total divided into phil deep and shallow nerve. Hoang NS [7] the results showed that the curative ring GB34 motor 
nerve injury of sciatic nerve compression model can promote the recovery of function. Qu M [8] clinical study showed that acupuncture GB34 acupoints on the waist dish outstanding to cause of sciatica has good curative effect. The analgesic effect of CCI + EA group is superior to the CCI +5 aza group $(\mathrm{P}<0.05)$, CCI + EA group Dnmt3amRNA expression was slightly higher than that of CCI +5 aza group, but no significant difference $(\mathrm{P}>0.05)$.Thus, we consider electric acupuncture ST36, GB34 inhibition of spinal dorsal horn Dnmt3a mRNA expression, to achieve the effect of analgesia.

Above all, DNA methylation and closely related to chronic pain, electric acupuncture ST36, GB34 can alleviate pain, and can adjust Dnmt3a mRNA expression. Electric acupuncture ST36, GB34 may be by reducing spinal cord dorsal horn overall level of methylation, reach the role of analgesia.

Author Contributions: Qiu-ling XU was responsible for animal experiments, data analysis and drafting of the manuscript in Chinese and English, designed the study and provided research funding. Tao Liu was responsible for the data analysis and figures making, Tao Liu is the equal contribution with Qiu-ling XU .

Funding: This work was supported by the National Natural Science Foundation of China, No. 81360560,No.81660820

\section{References}

[1] Denk F, McMahon SB. Neuron. 2012. 73(3): 435-44.

[2] Wang Y, Liu C, Guo QL, et al. Brain Res. 2011. 1418: 64-9.

[3] Sun Y, Sahbaie P, Liang D, et al. PLOS ONE. 2015. 10(11): e0142046.

[4] Abzianidze E, Kvaratskhelia E, Tkemaladze T, Kankava K, Gurtskaia G, Tsagareli M. Georgian Med News. 2014. (235): 78-81.

[5] Wang Y, Guo Q, Yan J, Yang M, Lin Y, Lin Z.Zhong Nan Da Xue Xue Bao Yi Xue Ban. 2016 Jun 28;41(6):578-85

[6] Pollema-Mays SL, Centeno MV, Apkarian AV, Martina M.Front Cell Neurosci. 2014 Aug 8;8:217

[7] Hoang NS, Sar C, Valmier J, Sieso V, Scamps F. BMC Complement Altern Med. 2012. 12: 141.

[8] Qu M, Ding XN, Liu HB, Liu YQ. Zhong-guo Zhen Jiu. 2010. 30(8): 633-6. 